Vittalle - Revista de Ciências da Saúde v. 31, n. 1 (2019) 36-43
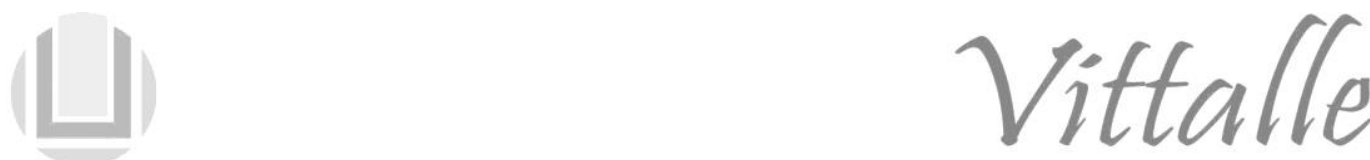

\title{
Ações de enfermagem em cuidado paliativo: conhecimento dos estudantes de graduação
}

\author{
Diego Augusto Lopes Oliveira*, Nayale Lucinda Andrade Albuquerque, \\ Maria Eduarda Carneiro Ramos, Rafaela Camboim Catão, Natalia do Nascimento Santos \\ Curso de Graduação em Enfermagem, Centro Universitário Tabosa de Almeida (ASCES/UNITA), Caruaru, PE, Brasil
}

Histórico do Artigo

Recebido em:

04/01/2019

Aceito em:

06/03/2019

\section{Palavras-chave:}

Educação em

Enfermagem;

Bacharelado em

Enfermagem; Equipe de

Enfermagem;

Enfermagem; Cuidado

Paliativo.

Keywords:

Nursing Education;

NursingBaccalaureate;

Nursing

Team;Palliative Care.

\begin{abstract}
RESUMO
Objetivou-se identificar o conhecimento do estudante sobre as ações do enfermeiro no desenvolvimento dos cuidados paliativos. Trata-se de um estudo descritivo de abordagem qualitativa onde foi aplicado roteiro de entrevista entre estudantes de um curso de graduação em enfermagem do interior de Pernambuco. As entrevistas foram gravadas e realizadas mediante consentimento formal do sujeito de pesquisa e autorização do comitê de ética na pesquisa. A partir dos relatos foram encontradas a seguintes categorias de análise: Papel do enfermeiro como essencial no processo do cuidado na finitude; Esclarecer e comunicar-se com o paciente em processo de finitude; Trabalhar em equipe multiprofissional; Realizar um cuidado integral e continuado. Observou-se que os 11 estudantes entrevistados apresentam compreensão satisfatória do papel do profissional enfermeiro destacando uma prática pautada nos ideais de humanização, comunicação, parceria com outros profissionais e no cuidado integral e continuado. O perfil de formação que oportuniza a vivência no referencial de cuidados paliativos possibilita maior entendimento sobre a modalidade de atenção e leva o acadêmico a desenvolver novas frentes de atenção na oferta de experiências de cuidado mais satisfatórias para o paciente e familiares.
\end{abstract}

Nursing actions in palliative care: knowledge of graduation students

\begin{abstract}
The objective was to identify the student's knowledge about the actions of the nurse in the development of palliative care. This is a descriptive study of a qualitative approach, where an interview script was applied among students of a nursing undergraduate course in the interior of Pernambuco. The interviews were recorded and performed by formal consent of the research subject and authorization of the Research Ethics Committee. From the reports, the following categories of analysis were found: role of the nurse as essential in the process of care in finitude; Clarify and communicate with the patient in the process of finitude; Work in a multiprofessional team; Complete and continued care. It was observed that the 11 students interviewed presented satisfactory comprehension of the role of the nurse professional highlighting a practice based on the ideals of humanization, communication, partnership with other professionals and in integral care and Continued. The formation profile that opportunites the experience in the referential of palliative care allows a greater understanding about the modality of care and leads the scholar to develop new attention fronts in the provision of more satisfactory care experiences for Patient and family.
\end{abstract}

\section{Introdução}

O termo "cuidados paliativos" é utilizado para designar a ação de uma equipe multiprofissional a pacientes fora de possibilidades terapêuticas de cura. A palavra "paliativo" é originada do latim pallium que significa manto, proteção, ou seja, proteger aqueles em que a prática curativa já não mais acolhe (1). Esses cuidados são ativos e totais ao paciente cuja doença não responde mais ao tratamento curativo. Trata-se de uma abordagem diferenciada que visa melhorar a qualidade de vida do paciente e seus

\footnotetext{
*Autor correspondente: diegoaugusto.enf@gmail.com (Oliveira D.A.L)
} 
familiares, por meio da adequada avaliação e tratamento para alívio da dor e sintomas, além de proporcionar suporte psicossocial e espiritual (2).

Baseada em uma visão holística do ser humano esses cuidados têm como filosofia valorizar a vida e encarar a morte como um processo natural. Assim, não adia e nem prolonga a morte, mas ampara o ser em suas angústias e medos, oferecendo suporte para que os pacientes possam viver o mais ativamente possível, ajudando a família e os cuidadores diante do processo de luto (3).

A enfermagem, por ser uma profissão que está em contato direto com o paciente, é responsável por um olhar holístico que contempla o processo de cuidar nas dimensões biológica, psicológica, social e espiritual do ser humano (4). Na implementação desses cuidados há necessidade da intervenção de uma equipe de profissionais adequadamente treinados e experientes no controle de sintomas de natureza não apenas biológica, mas também com excelente potencial de comunicação, para que o paciente e seus familiares entendam o processo evolutivo que atravessam, tenham o conhecimento da história natural da doença em curso, para que se possa atuar de forma a proporcionar não apenas o alívio, mas a prevenção de um sintoma ou situação de crise (5).

Atualmente o ensino dessa modalidade de atenção é pouco difundido e abordado na graduação em saúde/enfermagem. O pouco conhecimento e vivência do estudante acerca do manejo destes pacientes culmina numa finalização da graduação sem preparo para suporte aos indivíduos e seus familiares (6).

Nesses pressupostos objetiva-se com este estudo identificar o conhecimento do estudante sobre as ações do enfermeiro no desenvolvimento dos cuidados paliativos.

\section{Materiais e métodos}

Trata-se de um estudo descritivo de abordagem qualitativa, realizado com discentes do $9^{\circ}$ módulo de enfermagem de uma instituição de ensino superior da cidade de Caruaru-PE.

No referido curso de graduação, com currículo integrado, os estudantes vivenciam a unidade temática nomeada "Práticas de Assistência Domiciliar" na qual desenvolvem atividades teórico práticas voltadas a compreensão do papel do profissional enfermeiro na assistência ao paciente em cuidados paliativos. Nas referidas atividades o estudante tem a oportunidade de visitar serviços referência nesta modalidade assistencial como forma de contribuir na construção dos seus conhecimentos. O curso é pioneiro na sua região na oferta deste referencial em uma graduação em enfermagem, estando alinhado às práticas atuais a pacientes crônicos e as necessidades pontuadas pelos órgãos especializados.

A coleta foi realizada no mês de dezembro de 2017, por meio de entrevistas utilizando-se de um roteiro semiestruturado elaborado pelos pesquisadores, que após o aceite dos discentes, assinaram o Termo de Consentimento Livre e Esclarecido (TCLE).

Para operacionalização da coleta dos dados foi promovido momento com os estudantes para orientações acerca do estudo e considerados como critérios de inclusão a fidelização da matrícula do estudante no curso de graduação em enfermagem da referida instituição e a vivência obtida na unidade temática relacionada às práticas de assistência domiciliar.

Os relatos dos estudantes foram gravados durante uma abordagem em uma sala de aula da própria instituição e imediatamente transcritos para aplicação do critério de saturação amostral (7). A partir da aplicação deste pressuposto foram considerados 11 estudantes como componentes da amostra, cujas entrevistas foram codificadas com a letra E de "entrevistado", seguida dos números de 1 a 11. Utilizada como questão norteadora: "No seu entendimento qual o papel do profissional de enfermagem na assistência ao paciente em cuidados paliativos?".

A análise de conteúdo foi à técnica de Bardin, desenvolvida para sistematização dos dados, sendo aplicadas nas seguintes etapas metodológicas: $1^{\text {a }}$ etapa, pré-análise, em 
que foram organizados os depoimentos e a constituição do corpus de análise; a $2^{\mathrm{a}}$ etapa compreendeu a exploração do material; a $3^{a}$ etapa consistiu no tratamento dos dados, a partir da frequência de presença e verificação da homogeneidade de sentido (8).

O estudo foi aprovado pelo Comitê de Ética em Pesquisa do Centro Universitário Tabosa de Almeida sob o CAAE 106 79716117.0.0000.5203.

\section{Resultados}

Os 11discentes do $9^{\circ}$ módulo de enfermagem que participaram da pesquisa tinham idade entre 20 e 25 anos, onde 8 eram do sexo do feminino e 3 do sexo masculino. Estes não prestavam serviços em ambientes que oportunizassem contato direto com pacientes indicados a paliação ou em processo de finitude. Após a análise de conteúdo das falas, emergiram quatro categorias temáticas: "Papel essencial do Enfermeiro no processo de finitude"; "Responsabilidade em ofertar informações ao paciente em processo de finitude"; "Trabalhar em equipe multiprofissional"; "Realizar um cuidado integral".

\subsection{Papel do Enfermeiro é essencial no processo do cuidado na finitude}

Destacou-se, nos depoimentos dos participantes, o reconhecimento da importância do papel do Enfermeiro no cuidado ao paciente em processo de finitude. Este reconhecimento perpassa o entendimento do estudante quanto a uma das principais características desta profissão: o cuidado.

\footnotetext{
"O papel da enfermagem é essencial, né? É fundamental, visto que o enfermeiro ... é o responsável pelo cuidado, principalmente no processo de finitude." (E1)
}

Os estudantes ainda referem que o Enfermeiro é importante por ser o profissional mais próximo do paciente. É quem está ao lado, cuidando e observando detalhes da evolução do paciente durante todo o processo de atenção e é o principal ator na transmissão das informações quanto aos cuidados, exames, intercorrências e evoluções do mesmo a toda a equipe de cuidado.A humanização do cuidado de Enfermagem também foi considerada como essencial para a assistência ao paciente.

\footnotetext{
"Acho que o enfermeiro, ele tem uma extrema importância nesse sentido, porque por vezes é ele quem estabelece, assim, uma relação mais próxima com o paciente ..." (E2)

"É de grande importância, pode-se dizer fundamental, proporcionar ao paciente uma assistência qualificada, humanizada, garantindo a este uma qualidade de morte, uma morte digna. Servir como apoio para o paciente, bem como aos seus familiares." (E11)

“... ele (o enfermeiro) deve ser um profissional humanizado pra saber lidar com as dificuldades daquele paciente." (E9)

"É ofertar ao paciente o que ele necessita, escutá-lo. É atender suas exigências, respeitar o seu processo de finitude, tentar diminuir as angústias vividas pelo paciente." (E10)
}

\subsection{Esclarecer e comunicar-se com o paciente em processo de finitude}

Para os entrevistados, o Enfermeiro tem como atribuição importante propiciar informações claras ao paciente, sendo um importante profissional responsável pela comunicação com o paciente e familiares. A comunicação emerge como um fator 
importante para o esclarecimento dos direcionamentos terapêuticos e meio de aproximar o paciente à equipe de Enfermagem.

$$
\begin{aligned}
& \text { “... esclarecer tudo pra o paciente, sempre repassando, né?” (E6) } \\
& \text { “. ... dá esse direito a esse paciente ... implementar uma abordagem } \\
& \text { diferenciada, porque não é fácil, né? Você ser paliado. E o profissional de } \\
& \text { enfermagem, ele pode contribuir bastante na compreensão de toda essa } \\
& \text { paliação por parte do paciente ...” (E7) }
\end{aligned}
$$

\title{
3.3. Trabalhar em equipe multiprofissional
}

Os estudantes entrevistados reconhecem que existe uma grande contribuição ao paciente quando o Enfermeiro tem uma atitude de abertura para o trabalho em equipe multiprofissional, conforme observa-se na fala a seguir:

\begin{abstract}
"Então, o cuidado paliativo ele não depende não só da equipe de Enfermagem, mas multidisciplinar, né? Porque muitas vezes, por exemplo, cuidados básicos, como por exemplo, curativo e medicação, sim. Depende do enfermeiro. Mas precisa também do médico, do nutricionista, certo? ... depende de uma equipe multiprofissional, não só da enfermagem." (E8)
\end{abstract}

\subsection{Realizar um cuidado integral e continuado}

Nesta categoria, os entrevistados compreendem que o paciente, nesta fase, necessita que o profissional de saúde possa o considerar em todo o seu contexto e necessidade. $\mathrm{O}$ cuidado integral surge como um fator que permite qualidade de vida.

\footnotetext{
“... essas pessoas que estão ... em processo de finitude, elas precisam muito desses cuidados e um cuidado de forma geral, né? Visando sempre o paciente como um todo." (E3)

"Ele deve prezar pela qualidade de vida do paciente, ele deve realizar um cuidado integral..." (E4)
}

Além de ressaltar o cuidado integral, foi identificada a necessidade de promover a continuidade das ações assistenciais, permitindo que o cuidado desenvolvido junto ao paciente em finitude não tivesse quebra. A implementação das ações é demonstrada de forma em que a equipe mantenha relacionamento e comunicação em prol da manutenção do cuidar de forma harmoniosa e com promoção de benefícios ao paciente.

“... no desenvolvimento do cuidado continuado, visando ... buscar o
conforto e bem estar do paciente que necessita de uma assistência ampla,
buscando uma melhora não só física, mas também espiritual.” (E5)

\section{Discussão}

A enfermagem identifica-se fundamentalmente por meio do cuidado. Este se legitima como objeto epistemológico. Sendo assim, as instituições de ensino e os profissionais da prática aprimoram-no, considerando-o a própria essência da profissão. Esse domínio de conhecimento lhe confere a competência do cuidar dos seres humanos em todas as dimensões do processo de viver (9).

Assistir o doente nas suas necessidades básicas define a enfermagem como ciência e arte, em se tratando de cuidados paliativos, pode-se acrescentar que essa profissão busca contribuir para uma sobrevida mais digna e uma morte tranquila através da utilização de evidências para fortalecimento das ações de cuidado a serem desenvolvidas garantindo benefícios ao indivíduo e família (10). 
A enfermeira é quem geralmente está próximo nos momentos difíceis, é quem o paciente e a família buscam quando necessitam de esclarecimentos, ou de cuidados imediatos. Assim este profissional tem que lidar com o sofrimento, com a angústia e com os temores que podem surgir em diversas situações que envolvem esse cuidar (11). Sendo assim, a comunicação é extremamente relevante na relação terapêutica que se estabelece entre a equipe e o paciente, e tem por finalidade proporcionar confiança e reduzir a ansiedade para que se possa alcançar uma relação de ajuda efetiva, na qual o paciente e sua família possam expressar temores, angústias, valores e significados (12).

A compreensão dos estudantes no tocante a linha de comunicação entre profissional de enfermagem e paciente em paliação é adequada, demonstrando que os aspectos verbais e não verbais possibilitam uma maior compreensão do profissional das necessidades do paciente e instrumentalização de uma prática voltada a objetivos reais e individuais.

Após o lançamento do Programa Nacional de Humanização, existe uma atenção maior quanto ao acolhimento, o programa tem como um dos objetivos capacitar os profissionais para um novo conceito de assistência à saúde que valorize a vida humana $\mathrm{e}$ a cidadania (13). A Humanização da assistência pode ser considerada a essência do processo de Enfermagem, já que o toque, a responsabilidade em transmitir informações, em garantir privacidade, em garantir assistência baseada em evidências científicas, em escutar o paciente faz parte de sua rotina de cuidados.

Para cuidar de forma humanizada, o profissional da saúde, principalmente o enfermeiro, que presta cuidados mais próximos ao paciente, deve ser capaz de entender a si mesmo e ao outro, ampliando esse conhecimento na forma de ação e tomando consciência dos valores e princípios que norteiam essa ação. Neste contexto, respeitar e preservar a dignidade do paciente é componente primordial no tocante a cuidados humanizados (14).

Quando, na fala, é identificada a importância do escutar, de atender as necessidades do paciente e respeitar seu processo, revela-se aqui a importância da empatia na assistência pelos profissionais de saúde. O entendimento das ações de humanização da assistência pelos estudantes participantes da pesquisa vem proporcionar ao paciente a garantia de uma assistência com empatia e estimula ampliação do olhar relativo ao cuidado, ressignificando sua prática.

A assistência de enfermagem no contexto dos cuidados paliativos deve considerar o paciente um ser único, complexo e multidimensional: biológico, emocional, social e espiritual. Este tipo de cuidado, integral e humanizado, só é possível quando o enfermeiro faz uso de diversidades de comunicação para que perceba, compreenda e empregue a comunicação verbal e não-verbal (15).

No âmbito da Enfermagem, a comunicação representa uma estratégia de suma relevância para a prática dos cuidados paliativos. E quando subsidiada por uma relação de atitude, cooperação, sentimento e sensibilidade, este instrumento é um importante impulsionador da relação entre o enfermeiro e o paciente em fase terminal (16).

Compreende-se comunicação interpessoal na área de saúde e em cuidados paliativos como um processo complexo que envolve a percepção, a compreensão e a transmissão de mensagens na interação entre pacientes e profissionais de saúde. É um processo que possui duas dimensões: a verbal, que ocorre por meio da expressão de palavras faladas ou escritas; e a não verbal, caracterizada pelo jeito e tom de voz com que palavras são ditas, por gestos que acompanham o discurso, por olhares e expressões faciais, pela postura corporal, pela distância física que as pessoas mantêm umas das outras (16).

Nesse sentido, o enfermeiro deve comunicar-se efetivamente com o paciente, ouvindoo sempre que possível, de maneira que possa ajudar a pessoa a revelar os sentimentos que cercam o processo de terminalidade e luto, para que se possa compreender melhor a sua experiência (17). 
Além disso, existe uma compreensão de que o Enfermeiro possui habilidade para uma comunicação clara e que isto é um direito do paciente. O paciente que vivencia esta fase poder sentir-se acolhido, a partir do momento que compreende o que está acontecendo com ele mesmo, propicia sensação de segurança e possibilita decisões sobre questões pessoais da sua vida, além de planejamento conjunto quanto cuidado que o mesmo necessita.

O uso de estratégias comunicacionais é o centro do suporte emocional em cuidados paliativos. Habilidades para a escuta, questionamento, exploração de sentimentos e feedback não são uma questão de personalidade ou instinto do profissional, mas ferramentas necessárias para prover conforto emocional e influenciam positivamente $o$ ajuste psicológico do indivíduo à vivência do processo perdas e incertezas inerentes ao adoecimento e morte (18). Nesse sentido a comunicação deve ser disponível sem empreender ações diagnósticas ou terapêuticas inúteis ou obstinadas, levando sempre em consideração a vontade expressa do paciente ou, na sua impossibilidade, a de seu representante legal.

Para que o cuidado se desenvolva de forma integral e humanitária, o enfermeiro deve atender às necessidades de seus clientes/pacientes e de seus familiares, interagindo com eles, resolvendo problemas, apontando soluções, propiciando melhora da sua condição de saúde, ou proporcionando uma morte digna e com serenidade (19).

A abordagem complexa proposta pelos cuidados paliativo objetiva atender todas as dimensões do ser cuidado e de sua família, priorizando assim de uma equipe multiprofissional, que deve ser composta por enfermeiro, psicólogo, médico, assistente social, farmacêutico, nutricionista, fisioterapeuta, fonoaudiólogo, terapeuta ocupacional, cirurgião dentista e assistente espiritual. Entretanto, para alcançar esse objetivo, torna-se fundamental que o profissional adote uma postura reflexiva em relação às práticas de cuidado, de modo que as instituições hospitalares visem à dignidade e totalidade do ser humano (20).

Nesta conjuntura, os profissionais devem atuar em todas as dimensões humanas no sentido de aliviar o sofrimento e confortar os pacientes e suas famílias, com a finalidade de obter uma melhor qualidade de vida. Portanto, cuidar na perspectiva paliativa consiste em garantir uma atenção holística, humana e individual para o paciente e sua família, a fim de transformar o processo de morte em uma situação menos árdua para as pessoas envolvidas (21).

Cuidar em enfermagem paliativa é prover conforto, agir e reagir adequadamente frente a situação de morte com o doente, família e consigo mesmo; é promover o crescimento pessoal do doente, família e de si mesmo, é valorizar o sofrimento e as conquistas, empoderar o outro com seu cuidado e empoderar-se pelo cuidado, é lutar para preservar a integridade física, moral, emocional e espiritual, é conectar-se e vincular-se e auxiliar o outro e a si mesmo a encontrar significados nas situações. Cuidar em enfermagem paliativa é prover o alívio de sintomas, ser flexível, ter objetivos de cuidado, advogar pelo doente e reconhecê-lo como ser humano único (22).

Os papéis do enfermeiro em cuidados paliativos são educar, cuidar, promover, advogar e coordenar e os atributos para exercer tais papéis são ter "expertise" clínica, manter o foco no doente e família, ter atos deliberados, intencionais, de cooperação, manter a honestidade na comunicação e estar presente, disponível e atento (discernir com sabedoria) (22).

Para a assistência integral que a fase dos cuidados paliativos requer, faz-se necessária uma formação acadêmica que contemple esta temática. Entretanto, a literatura científica aponta que na formação dos profissionais de saúde, ainda há predomínio da lógica biologicista. As evidências científicas sobre a necessidade de preparar o graduando para 
enfrentar a morte mostram que os currículos nas instituições de ensino superior na área da saúde ainda não têm assegurado a contextualização da temática de modo consistente (23). Para que os futuros profissionais tenham uma visão humanística acerca das necessidades dos pacientes fora de possibilidades terapêuticas de cura, é necessário que haja uma modificação no currículo dos cursos de graduação, privilegiando-se conteúdos específicos sobre os cuidados paliativos (6).

\section{Considerações finais}

O conhecimento expresso pelos estudantes de graduação em enfermagem possibilita uma reflexão aprofundada sobre a vivência do referencial temático de cuidados paliativos nos cursos de graduação, ficando evidente a relação entre essa modalidade de atenção e a essência da atividade do Enfermeiro, evidenciando avanços e bons resultados no manejo da doença e nas relações entre profissional, indivíduo e família.

Essa ainda é uma temática pouco abordada e vivenciada nas grades curriculares, por esse aspecto há um despreparo dos profissionais no momento da prestação desses cuidados, sendo estes fragmentados dificultando assim uma assistência integral e continuada. A vivência e compreensão deste referencial temático oportuniza uma potencialização da qualidade do serviço prestado pela categoria e agrega, ao graduando, conhecimentos imprescindíveis para uma prática pautada nos princípios norteadores dessa modalidade de atenção. $\mathrm{O}$ desenvolvimento desta temática na graduação ainda é pequena e não reflete o panorama das instituições de ensino superior de enfermagem no país.

A implementação do ensino deste referencial na instituição de ensino referida evidencia novas dimensões e perspectivas na atenção a pacientes em cuidados paliativos oportunizando novos esforços, não só dos profissionais de enfermagem, para atenção adequada e humanizada. Observou-se como limitação deste estudo o número restrito de estudantes que vivenciaram a referida unidade temática para desenvolvimento da pesquisa.

Vislumbra-se como importante o desenvolvimento de futuros estudos voltados à compreensão da percepção dos estudantes de curso de graduação em saúde para a modalidade dos cuidados paliativos de forma multiprofissional e como componente curricular nos referidos cursos superiores.

\section{Referências}

1. Hermes HR, Lamarca ICA. Cuidados paliativos: uma abordagem a partir das categorias profissionais de saúde. Ciência \& Saúde Coletiva 2013;18(9): 2577-88.

2. World Health Organization (WHO). WHO Definition of Palliative Care [Internet]. Geneva: 2010 [citado em 2018Dec26]. Disponível em: https://www.who.int/cancer/palliative/definition/en/.

3. Bertachini L, Pessini L. A importância da dimensão espiritual na prática dos cuidados paliativos. Rev Centro Universitário São Camilo 2010;4(3):315-323.

4. Nascimento LC, Santos TFM, Oliveira FCS, Pan R, Flória-Santos M, Rocha SMM. Espiritualidade e religiosidade na perspectiva de enfermeiros. Texto Contexto Enferm 2013; 22(1):52-60.

5. Foley KM. The past and the future of palliative care. Improving end life care: why has it been so difficult? Hastening Center Report 2005; 35(6):01-06.

6. Duarte AC, Almeida DV, Popim RC. A morte no cotidiano da graduação: um olhar do aluno de medicina. Interface (Botucatu) 2015; 19(55): 1207-1219.

7. Fontanella BJB, Luchesi BM, Saidel MGB, Ricas J, Turato ER, Melo DG. Amostragem em pesquisas qualitativas: proposta de procedimentos para constatar saturação teórica. Cad Saúde Pública 2011; 27(2): 389-394.

8. Bardin L. Análise de conteúdo. Lisboa: Edições 70; 2011.

9. Pires D. A enfermagem enquanto disciplina, profissão e trabalho. RevBrasEnferm 2009; 62(5):739-44. 
10. Matos FA, Moraes TM. A Enfermagem nos cuidados paliativos. In: Figueiredo MTA. Coletânea de textos sobre cuidados paliativos e Tanatologia. São Paulo: Unifesp; 2006. p. 49-62.

11. Machado WCA, Leite JL. Eros e Thanatos: a morte sob a óptica da enfermagem. São Caetano do Sul: Yends; 2006.

12. Melo AGC, Caponero R. O futuro em cuidados paliativos. In: Santos FS. Cuidados paliativos: diretrizes, humanização e alívio dos sintomas. São Paulo: Atheneu; 2011.

13. Política Nacional de Humanização- PHN [internet]. Brasília: Ministério da Saúde [Atualizado em 2013; citado em 2018 Dec 27]. Disponível em: http://bvsms.saude.gov.br/bvs/publicacoes/politica_nacional_humanizacao_pnh_folheto.pdf .

14. Pessini L. Humanização da dor e do sofrimento humanos na área da saúde. In: Pessini L, Bertachini L. Humanização e cuidados paliativos. São Paulo: Loyola; 2004.

15. Araújo MMT, Silva MJP. A comunicação com o paciente em cuidados paliativos: valorizando a alegria e o otimismo. RevEscEnferm USP 2007; 41(4): 668-74.

16. Mullan BA, Kothe EJ. Evaluating a nursing communication skills training course: the relationships between self-rated ability, satisfaction, and actual performance. Nurse EducPract2010; 10(6): 374-8

17. Lopes MEL, Fernandes MA, Platel ICS, Moreira MADM, Duarte MCS, Costa TF.Palliative care: understanding of the assistant nurses. RevEnferm UFPE 2013;7(1):168-175.

18. Muniz RM, Zago MMF, Schwatz E. As teias da sobrevivência oncológica: com a vida de novo. Texto Contexto Enferm 2009; 18(1): 25-32.

19. Costenaro RGS, Lacerda MR. Quem cuida de quem cuida, quem cuida do cuidador. Santa Maria: Centro Universitário Franciscano; 2002.

20. Academia Nacional de Cuidados Paliativos. Manual de cuidados paliativos. Rio de Janeiro: Diagraphic; 2009.

21. Araújo DF, Barbosa MH, Zuffi FB, Lemos RCA. Cuidados paliativos: percepção dos enfermeiros do Hospital das Clínicas de Uberaba-MG. RevCiência, Cuidado e Saúde2010; 9(4): 690-696.

22. Pimenta CA. Palliative care: a new specialty in profession of nursing?Acta Paul Enferm 2010; 23(3): 01-02.

23. Souza LF, Misko MD, Silva L, Poles K, Santos MR, Bousso RS. Dignified death for children: perceptions of nurses from an oncology unit. RevEscEnferm USP 2013; 47(1):30-7. 